\title{
Assessment of vascularized free fibula transplantation revealing a congenital aplastic posterior tibial artery: a case report
}

Takako Kanatani $^{1 *}$, Issei Nagura', Ikuo Fujita ${ }^{2}$, Takuya Fujimoto ${ }^{2}$ and Masatoshi Sumi ${ }^{1}$

\begin{abstract}
Introduction: Anatomical abnormalities in the lower limb vessels are uncommon. However, the preoperative evaluation of the anatomical variations is very important for planning the operation procedure to prevent jeopardizing the donor leg.
\end{abstract}

Case presentation: In this case report, a 23-year-old Asian woman who was scheduled to have vascularized free fibula transplantation for reconstruction of her wrist after excision of bone tumor in her distal radius, was found to have congenital aplastic posterior tibial arteries in both legs. These findings were found on magnetic resonance angiography (our preferred methodology due to its simplicity). We planned testing the sufficiency of her pedal pulses after temporarily clamping her peroneal artery but prior to harvesting, to ensure minimal risk to the longevity of her donor leg. During the operation, after dissection of a $10 \mathrm{~cm}$ segment of her fibula with the peroneal artery, the peroneal artery proximal to the graft was temporarily clamped and the tourniquet was released. As adequate sustainable pedal pulses were confirmed, the graft was harvested and transplanted to her wrist. There was no morbidity in her right leg postoperatively and the union of the grafted fibula was substantiated 10 months postoperatively.

Conclusions: We concluded two findings: firstly, for accurate preoperative planning of a vascularized free fibula procedure, examination of the bilateral lower leg vasculature either by angiography or other imaging should be performed. Secondly, abnormalities are not in themselves reason to abandon the vascularized free fibula procedure. We contend that pedal pulses should be evaluated preoperatively and provided that adequate foot circulation can be confirmed (by temporarily clamping the vessels and releasing the tourniquet during the operation prior to harvesting the free vascularized fibula) the procedure should be successful without jeopardizing the donor leg.

Keywords: Magnetic resonance angiography, Peroneal artery, Vascularized free fibula transplantation

\section{Introduction}

A vascularized free fibula transplantation is useful to reconstruct large segmental bone defects $(>6 \mathrm{~cm})$ of the upper extremity [1-3]. An anatomical assessment of bilateral lower leg vasculature either by angiography or magnetic resonance angiography (MRA) is recommended prior to a vascularized free fibula procedure [4-7]. Although anatomical abnormalities in the lower limb vessels are uncommon, angiographic abnormalities have been reported at the rate of $3 \%$ to $25 \%$ [4-6,8] in different

\footnotetext{
* Correspondence: takakokatie@hotmail.com

'Department of Orthopaedic Surgery, Kobe Rosai Hospital, 4-1-23,

Kagoike-dori, Chuo-ku, Kobe 651-0053, Japan

Full list of author information is available at the end of the article
}

situations. These showed variations of branching of the popliteal artery with only two or even one main artery supplying the foot with either the posterior tibial, the anterior tibial or both being hypoplastic-aplastic.

This report describes a successful vascularized free fibula transplantation to the wrist (reconstruction after the excision of bone tumor) in which the donor leg was not jeopardized, where a congenital aplastic posterior tibial artery (PTA) in the donor lower leg was detected.

\section{Case presentation}

A MRA of a 23-year-old Asian woman who was scheduled for a vascularized free fibula transplantation to reconstruct her left wrist after excision of a giant cell 
tumor in her distal radius (Figure $1 \mathrm{~A}$ and $\mathrm{B}$ ) showed a congenital aplastic PTA in her right lower leg (Figure 2). Her peroneal artery (PA) replaced the PTA at the medial malleolus and a similar finding was displayed in her left leg (Figure 2). In addition, her pedal pulses on her left foot were not palpable (due to previous knee trauma) making this fibula inappropriate as a donor section. In preoperative planning, a $10 \mathrm{~cm}$ segment of bone graft was required for partial wrist fusion. It is generally recognized that a foot survives with anterior tibial artery only without PTA if the pedal pulses are maintained. Therefore we planned to confirm the pedal pulses before harvesting the graft to prevent jeopardizing her lower leg. We advised her of the risks involved and explained the precaution of testing the sufficiency of her pedal pulses after temporarily clamping her PA prior to harvesting, to ensure minimal risk to the longevity of her donor leg. If it was found that there was insufficient palpability of her pedal pulses then the procedure would be aborted.

During the operation, after dissection of a $10 \mathrm{~cm}$ segment of her fibula with the PA, the PA proximal to the graft was temporarily clamped and the tourniquet was released. As adequate sustainable pedal pulses were confirmed, the graft was harvested and transplanted to her wrist (Figure 3). There was no morbidity in her right leg postoperatively and the union of the grafted fibula was substantiated 10 months postoperatively.

\section{Discussion}

Anatomical abnormalities in the lower limb vessels are uncommon. Strauch and $\mathrm{Yu}$ reported the anatomical variations of the PA and classified four different types [9]. According to their classification, our case is classified as Type D; the PA took the place of the PTA in 8\% of all cases. However, Pototschnig et al. analyzed the intraoperative variations of the PA of 104 cases and classified four variations [10] in which there was no case that presented as “Type D" by Strauch et al. Angiographic abnormalities have been variously reported at the rate of $5.6 \%$ [4], 15.8\% [5], 25\% [6] and 3\% [8] in different situations. These show variations of branching of the popliteal artery with only two or even one main artery supplying the foot with either the tibial posterior, the tibial anterior or both being hypoplastic-aplastic. Most reports

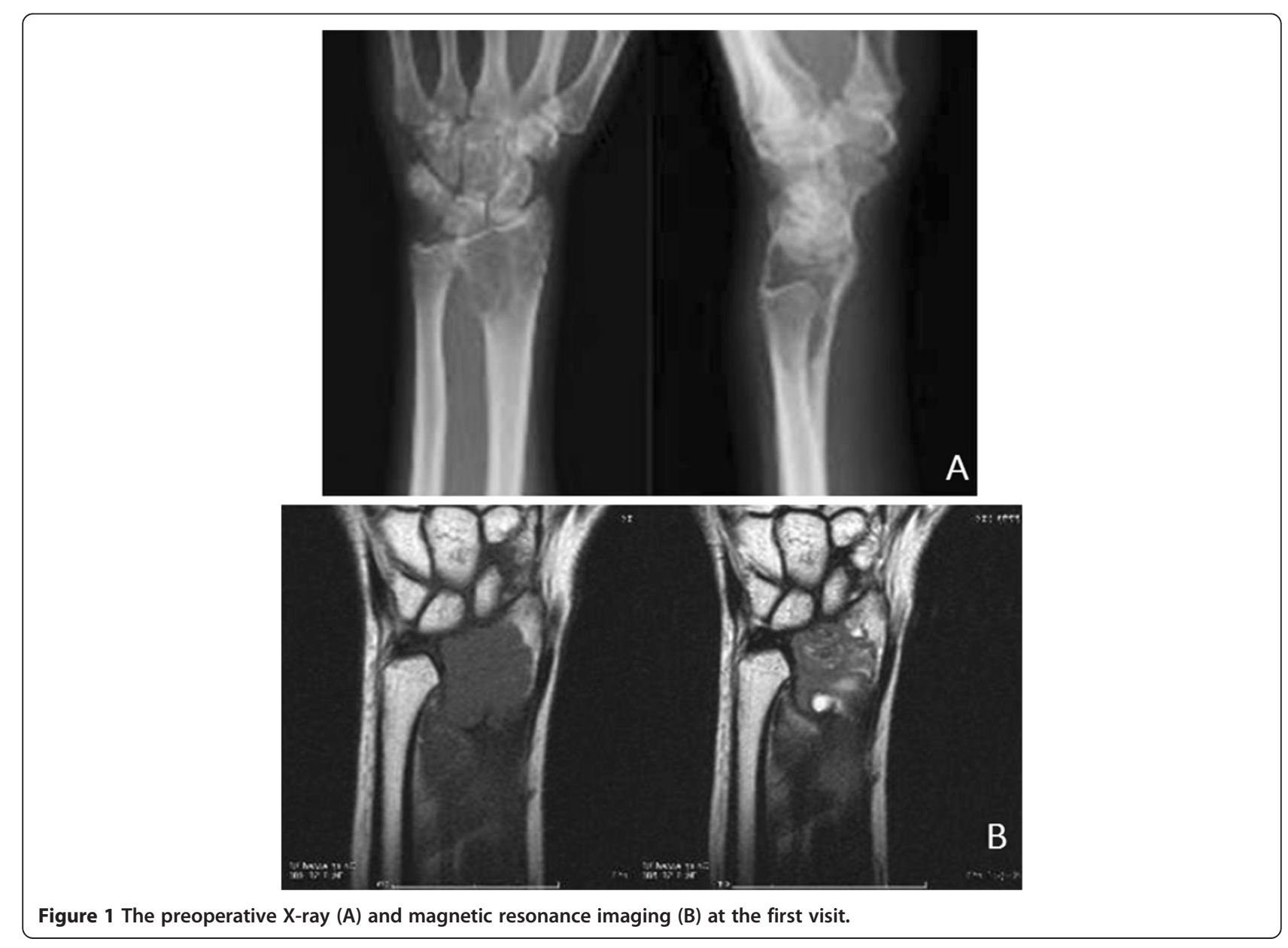




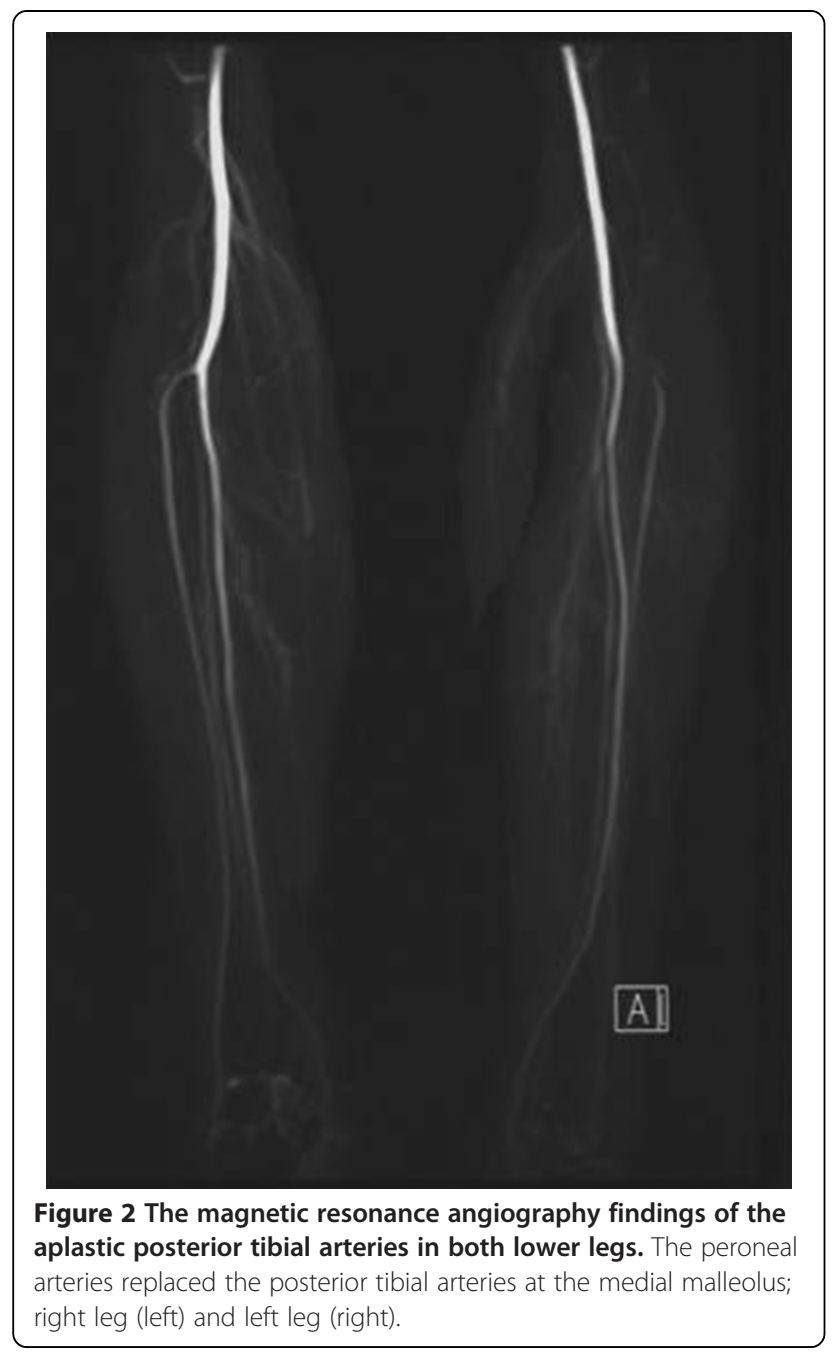

recommend a routine preoperative anatomical assessment of bilateral lower leg vasculature either by angiography, by MRA or by ultrasonic Doppler flow meter before a vascularized free fibula or fibula flaps procedure [4-7,9]. Young et al. altered the operation plan in all $25 \%$ of cases planned for fibula harvest where angiographic abnormalities were detected [6]. However, we consider preoperative angiographic abnormalities in themselves an insufficient reason to abandon the vascularized free fibula procedure. We note that Lutz et al. reported the possibility of a false positive angiography due to vascular spasms and they concluded that clinical evaluation of pedal pulses was superior to angiography as a diagnostic tool in these cases [8].

Regarding the choice of the operation procedure, wrist arthroplasty with an articular fibular head graft was an option. However, the long-term clinical and radiographic results of the partial wrist arthrodesis with fibula bone for giant cell tumors of the distal radius were superior to arthroplasty with an articular fibular head graft

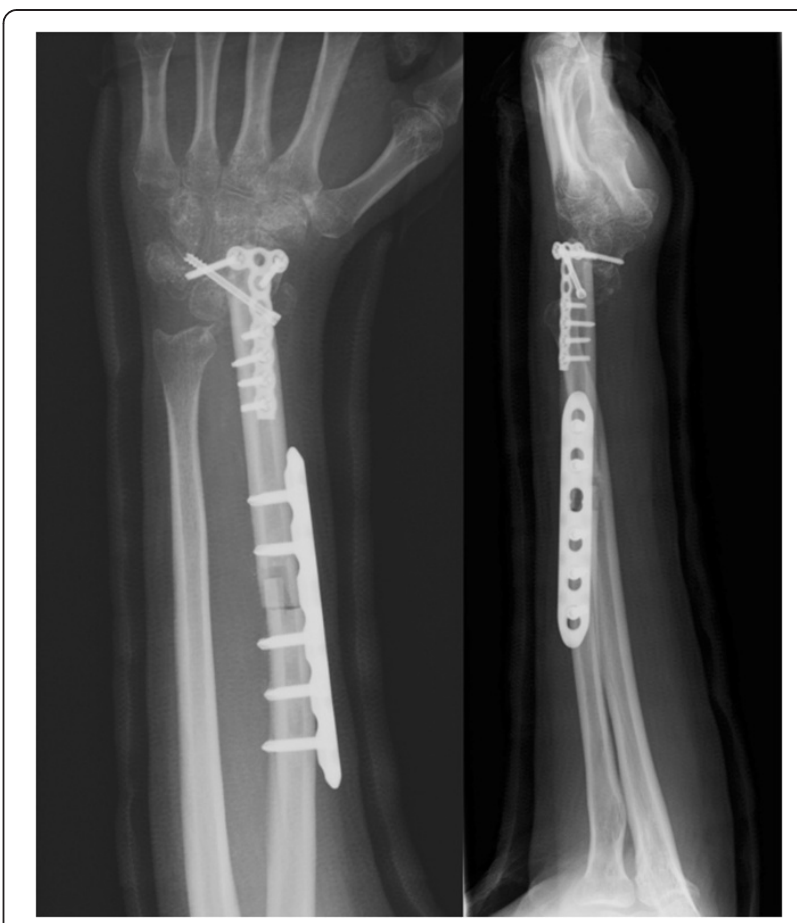

Figure $3 \mathrm{~A} 10 \mathrm{~cm}$ segment of fibula bone graft was used for partial wrist fusion.

[11], where wrist arthroplasty presented palmar subluxation of the carpal bones and degenerative changes in all cases. Also, Chung et al. reported that half of their cases presented those changes during the long-term follow-up period [12]. We selected the partial wrist arthrodesis because of the patient's age of 23 years.

\section{Conclusions}

Consequently we conclude two findings: firstly, that for accurate preoperative planning of a vascularized free fibula procedure, examination of the bilateral lower leg vasculature either by angiography or other imaging should be performed. We suggest MRA would be the preferred methodology due to its simplicity in providing the required data. Secondly, where abnormalities are detected, pedal pulses should be evaluated preoperatively. Then provided adequate foot circulation can be confirmed by temporarily clamping the vessels and releasing the tourniquet during the operation prior to harvesting the free vascularized fibula, the procedure should be successful.

\section{Consent}

Written informed consent was obtained from the patient for publication of this case report and accompanying images. A copy of the written consent is available for review by the Editor-in-Chief of this journal. 


\section{Abbreviations}

MRA: Magnetic resonance angiography; PA: Peroneal artery; PTA: Posterior tibial artery.

\section{Competing interests}

We declare that we have no competing interests.

\section{Authors' contributions}

TK and IN were major contributors in harvesting the vascularized free fibula and in writing the manuscript. IF and TF analyzed and interpreted the patient data and performed the excision of the tumor and bone fixation of the fibula. MS performed the analysis of MRA and magnetic resonance imaging. All authors read and approved the final manuscript.

\section{Acknowledgement}

The authors acknowledge the contribution of Warren McPherson PhD for his grammatical assistance with English.

\section{Author details}

'Department of Orthopaedic Surgery, Kobe Rosai Hospital, 4-1-23, Kagoike-dori, Chuo-ku, Kobe 651-0053, Japan. ${ }^{2}$ The Department of Orthopaedics of Hyogo Cancer Center, 13-70, Kitaoji-Cho, Akashi 673-8858, Japan.

Received: 22 July 2013 Accepted: 20 December 2013

Published: 26 February 2014

\section{References}

1. Hollenbeck ST, Komatsu I, Woo S, Schoeman M, Yang J, Erdmann D, Levin LS: The current role of the vascularized-fibular osteocutaneous graft in the treatment of segmental defects of the upper extremity. Microsurgery 2009, 31:183-189.

2. Levin LS: Vascularized fibula graft for the traumatically induced longbone defect. J Am Acad Orthop Surg 2006, 14:S175-S176.

3. Arai K, Toh S, Tsubo K, Nishikawa S, Narita S, Miura H: Complications of vascularized fibula graft for reconstruction of long bones. Plast Reconstr 2002, 109:2301-2306.

4. Kim D, Orron DE, Skillman JJ: Surgical significance of popliteal arterial variants: a unified angiographic classification. Ann Surg 1989, 210:776-781.

5. Seres L, Casaszar J, Voros E, Borbely L: Donor site angiography before mandibular reconstruction with fibula free flap. J Craniofac Surg 2001, 12:608-613

6. Young DM, Trabulsy PP, Anthony JP: The need for preoperative leg angiography in fibula free flaps. J Reconstr Microsurg 1994, 10:283-287.

7. Monaghan AM, Dover MS: Short communication assessment of free fibula flaps: a cautionary note. Br J Oral Maxillofac Surg 2002, 40:258-259.

8. Lutz BS, Wei FC, Ng S, Chen IH, Chen SH: Routine donor leg angiography before vascularized free fibula transplantation is not necessary: a prospective study in 120 clinical cases. Plast Reconstr Surg 1999, 103:121-127.

9. Strauch $B, Y u H L$ : Lower leg and knee: variations of the peroneal artery. In Atlas of Microvascular Surgery. Edited by Strauch B, Yu HL. New York: Thieme; 1993:219.

10. Pototschnig H, Schaff J, Kovacs L, Biemer E, Papadopulos NA: The free osteofasciocutaneous fibula flap: Clinical applications and surgical considerations. Injury 2013, 44:366-369.

11. Minami A, Kato H, Iwasaki N: Vascularized fibular graft after excision of giant cell tumor of the distal radius: wrist arthroplasty versus partial wrist arthrodesis. Plast Reconstr Surg 2002, 110:112-117.

12. Chung DW, Han CS, Lee JH, Lee SG: Outcomes of wrist arthroplasty using a free vascularized fibular head graft for Enneking stage II giant cell tumors of distal radius. Microsurgery 2013, 33:112-118.

doi:10.1186/1752-1947-8-75

Cite this article as: Kanatani et al: Assessment of vascularized free fibula transplantation revealing a congenital aplastic posterior tibial artery: a case report. Journal of Medical Case Reports 2014 8:75.

\section{Submit your next manuscript to BioMed Central and take full advantage of:}

- Convenient online submission

- Thorough peer review

- No space constraints or color figure charges

- Immediate publication on acceptance

- Inclusion in PubMed, CAS, Scopus and Google Scholar

- Research which is freely available for redistribution 